\title{
Child health in China in the Millennium Development Goal era
}

\author{
Bo Sun, ${ }^{1}$ Kun Liang, ${ }^{2}$ Bin $\mathrm{Yi}^{3}{ }^{3}$ Li Zhang ${ }^{4}$
}

${ }^{1}$ Laboratory of Neonatal Medicine of National Health and Family Planning

Commission, Departments of Pediatrics and Neonatology, Children's Hospital of Fudan University, Shanghai, China ${ }^{2}$ Departments of Pediatrics and Neonatology, First Hospital of Kunming Medical University, Kunming, Yunnan, China ${ }^{3}$ Department of Neonatology, Gansu Provincial Women and Children's Hospital (Gansu Provincial Maternity Hospital), Lanzhou, Gansu, China

${ }^{4}$ Department of Neonatology, Northwest Women and Children's Hospital (Shaanxi Provincial Maternity Hospital), Xi'an, Shaanxi, China

\section{Correspondence to}

Professor Bo Sun, Departments of Pediatrics and Neonatology, Children's Hospital of Fudan

University, and the Laboratory of Neonatal Medicine of National Health and Family Planning Commission Shanghai 201102, China; bsun@shmu.edu.cn

Received 27 August 2014 Revised 3 September 2014 Accepted 4 September 2014

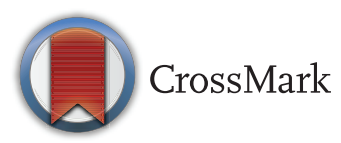

To cite: Sun $B$, Liang $K$, Yi B, et al. Arch Dis Child 2015;100(Suppl 1):s61-s62.
The Millennium Developmental Goal (MDG) 4, initiated in 1990 aiming to reduce mortality of children under 5 years of age (U5M) by two-third in 2015, was achieved in China to an average level lower than $15 / 1000$ in $2012^{1-3}$ (table 1). Given China's size (1.34 billion population with 16 million deliveries annually), this milestone has worldwide implications. This milestone was achieved through a combination of efforts: social, economic, governmental and medical. ${ }^{4}$ Over the two and half decades, U5M has undergone three phases of dramatic changes in China. First, the reduction of diarrhoea and pneumonia occurred between 1990 and 1999 as a result of enhanced public health policy in effect and living standard improvement when China was in transition from a low-income country towards a low-to-middle-income developing country, accounted for by improved public healthcare system through vaccination in infancy and childhood, housing and water supply, sanitation and essential healthcare.

Another prominent advance was significant reduction of maternal and infant mortality between 2000 and $2008,{ }^{3}{ }^{4}$ achieved by prenatal monitoring of high-risk pregnancy complication and in-hospital delivery, by eradication of neonatal tetanus and alleviation of birth asphyxia and by prevention and treatment of various neonatal diseases, including prenatal diagnosis and neonatal surgery. In general, in this period, there was rapid urbanisation so that maternal and infant healthcare was more efficient than that in rural areas in each province until 2010 when urban residents exceeded $50 \%$ of the total population. More very preterm infants survived from perinatal risks, ${ }^{5}$ and disparities of maternal and neonatal mortalities between rural and urban regions were narrowed from 4-5 times to 1-2 times (table 1), indicating the effectiveness of sustained policies and programme enforcement.

Finally, in 2009-2014, there was an introduction of widely established healthcare system, especially for nationwide rural residents subjected to $\mathrm{New}$ Rural Cooperative Medical Scheme to tackle availability and affordability of healthcare in neonate, infant and childhood in low-economic development provinces and regions. ${ }^{1}$ This universal healthcare insurance policy is based on voluntary participation for all rural families and individuals through payment of premium to achieve an average of 300-350 Chinese Yuan (CNY) per year per head, and national and provincial government finance will cover $80 \%-85 \%$ of it. This pool of health insurance collected is integrated to cover costs on stratified total sum and hospital service levels, reimbursing up to $70 \%-80 \%$ of the total patient hospitalised care costs, equivalent to 4-6 times of the local rural family annual total income.
Up to 2013, all the provinces and most of the rural residents were enrolled and subjected to this insurance. Adjustments were also made to enable those treated by provincial public healthcare services at non-resident registry area or eligible to special therapies for severe and chronic diseases to maximise the benefit for the rural participants. For newborn infants, their hospitalised costs are covered with their maternal insurance for up to $50 \%$ of the total sum.

In contrast to the cause of death of neonates and postneonatal infancy, the leading cause of U5M is associated with pulmonary infection (pneumonia) and sepsis, but unexpected asphyxia, traffic accident, near drowning, trauma and causes other than infection tended to become major ones impacting on paediatric emergency and intensive care. Congenital heart disease, tumour and malignancy, congenital malformation and genetically inherited metabolic disorders, and transmissible viral infection remain important morbidities contributing to U5M, which promoted establishment and improvement of paediatric surgical and paediatric critical care in almost all provincial and most subprovincial central hospitals. This was initiated by national and provincial special funds-based programmes to each county general or maternity hospitals of midland and west provinces to upgrade their paediatric critical care service standard that may serve for unexpected events of pandemics of severe systemic and respiratory viral infectious diseases and accidental traumatic hazard in children, in addition to daily care of neonatal and infant emergency needs.

Now, challenges for the post-MDG4 era will be the goal of the so-called 'women and children's healthcare plan', mainly at provincial and subprovincial levels by integrated efforts from public health and clinical medical care, social development and planning, finance and social welfare aspects, executed by the office from provincial commission of health and family planning (former provincial department of health) and its subsidiaries at subprovincial (city and regional) and county levels. The year 2014 is also designated as 'the year for women and children's healthcare service' by the National Health and Family Planning Commission ${ }^{1}$ to emphasise the importance of this plan through nationwide campaign. This plan aims to mitigate gaps between rural and urban areas by ensuring widely established monitoring and management of high-risk pregnancy through telemedicine, emergency transport and hospital delivery, thereby reducing maternal and neonatal death rate. This plan also promotes antenatal diagnostic and postdelivery recovery techniques, vaginal delivery and caesarean delivery restriction. 
Table 1 Maternal, neonatal, infant and under five children's mortality rate in 1991, 2005 and 2012 by national sampling surveillance system ${ }^{12}$

\begin{tabular}{lllcc}
\hline & Year & Total & Urban & Rural \\
\hline Maternal $(1 / 100000)$ & 1991 & 80 & 46.3 & 100 \\
& 2005 & 47.7 & 25.0 & 53.8 \\
Neonatal (1/1000) & 2012 & 24.5 & 22.2 & 25.6 \\
& 1991 & 33.1 & 12.5 & 37.9 \\
Infant $(1 / 1000)$ & 2005 & 13.2 & 7.5 & 14.7 \\
& 2012 & 6.9 & 3.9 & 8.1 \\
Children<5 years of age (1/1000) & 1991 & 50.2 & 17.3 & 58 \\
& 2005 & 19.0 & 9.1 & 21.6 \\
& 2012 & 10.3 & 5.2 & 12.4 \\
& 1991 & 61 & 20.9 & 71.1 \\
& 2005 & 22.5 & 10.7 & 25.7 \\
& 2012 & 13.2 & 5.9 & 16.2 \\
\hline
\end{tabular}

As neonatal death accounts for 50\%-60\% of U5M and almost $70 \%-75 \%$ of infant mortality, and more preterm neonates survive from initial birth-related diseases due to more effective perinatal intervention, there are several issues in daily neonatal and postneonatal care to be dealt with, such as how to restrict caesarean section from non-medical indication or low-risk pregnancy. Currently, nationwide average caesarean section rate should be around 35\%, 40\%-60\% in east provinces and major municipalities, but $20 \%-30 \%$ in many west provinces. How to keep increased very preterm infant survival with good quality of life is of importance in neonatal and developmental paediatric service. Composite preterm birth rate should be around $4 \%-5 \%$ of total births in both urban and rural areas. ${ }^{5}$ Very few data are published regarding gender ratio based on complete birth population from provincial and subprovincial regional vital statistics. ${ }^{5}$ High rate of parental give-up or withdrawal when treatment is effective in critically ill neonates and infants or concerns about long-term burden of worse prognoses in neurodevelopment are still challenges now.

With regards to limitations of the birth data through sampling of nationwide county and city district birth registry for vital statistics, many midland and west provinces started accumulating complete birth population-based annual reporting of these vital statistics as a part of the 'women and children's healthcare plan'. One of the most intriguing subjects is whether definition of perinatology should include those from 25 weeks (and up) of gestation, and perinatal death rate should be provided in the annual vital statistics regarding accurate registry of fetal death, stillbirth and early neonatal death. We found, in a regional complete birth registry-based survey, ${ }^{5}$ that perinatal and neonatal mortalities were associated with a cumulated 50\% survival rate in 29-30 weeks of gestation and in 1200-1300 g birth weight in contrast to that seen in the 23-25 weeks in developed countries.

To continue keeping U5M to a lower level, China's perinatalneonatal care is relying on, and interacting with, public health and paediatric service to ensure the improvement of community-based women and children's healthcare standard. We anticipate that, in the next 5-10 years with more concrete data coming out of the current practice and high-quality research, such improvement in the infrastructure for maternal and infant healthcare would show more conceivable progression, especially in rural regions of the west provinces.

Contributors BS organised the article content, references and concept, and wrote the manuscript; KL provided information regarding provincial women and children's healthcare plan and its perspectives in west provinces of China, and health insurance issue in perinatal care; BY provided information regarding provincial women and children's healthcare plan and its perspectives in west provinces of China, and high-risk pregnancy management information; LZ provided information regarding provincial women and children's healthcare plan and its perspectives in west provinces of China, and high-risk neonatal management and health insurance issue.

Funding Supported in part by Project 211 (phase III), Ministry of Education; the Project for Institutional Development in Laboratory of Neonatal Medicine, Ministry of Health; Shanghai Municipal Department of Health (no. LJ06038); and China Medical Board of New York (no. 03-786).

Competing interests None.

Provenance and peer review Commissioned; internally peer reviewed.

\section{REFERENCES}

1 State Council News Office of Peoples Republic of China. White book on medical and health care status in China, December 2012. http://www.nhfpc.gov.cn

2 National Health and Family Planning Commission. Yearbook of health statistics of China, July 2013. http://www.nhfpc.gov.cn

3 Feng J, Yuan XQ, Zhu J, et al. Under-5-mortality rate and causes of death in China, 2000 to 2010. Chin J Epidemiol 2012;33:558-61.

4 Feng $\mathrm{XL}$, Theodoratou E, Liu L, et al. Social, economic, political and health system and program determinants of child mortality reduction in China between 19902006: a systemic analysis. J Glob Health 2012;2:010405.

5 Sun L, Yue H, Sun B, et al. Estimation of birth population-based perinatal-neonatal mortality and preterm rate in China from a regional survey in 2010. J Matern-Fetal Neonat Med 2013;26:1641-8. 